\title{
VISUALIZING NETWORKS OF MUSIC ARTISTS WITH RAMA
}

\author{
Luís Sarmento (las@fe.up.pt) \\ LIACC / FEUP, Univ. do Porto, Rua Dr. Roberto Frias, s/n, Porto Portugal \\ Fabien Gouyon (fgouyon@inescporto.pt) \\ INESC Porto, Rua Dr. Roberto Frias, 378, Porto, Portugal \\ Bruno G. Costa (bgustavo@spymac.com) \\ Univ. Católica Portuguesa, Rua Diogo Botelho, 1327, Porto, Portugal \\ Eugénio Oliveira (eco@fe.up.pt) \\ LIACC / FEUP, Univ. do Porto, Rua Dr. Roberto Frias, s/n, Porto Portugal
}

\begin{abstract}
Keywords: Information visualization, Search interfaces, Content ranking using social media, User interfaces for search interaction

Abstract: $\quad$ In this paper we present RAMA (Relational Artist MAps), a simple yet efficient interface to navigate through networks of music artists. RAMA is built upon a dataset of artist similarity and user-defined tags regarding 583.000 artists gathered from Last.fm. This third-party, publicly available, data about artists similarity and artists tags is used to produce a visualization of artists relations. RAMA provides two simultaneous layers of information: (i) a graph built from artist similarity data, and (ii) overlaid labels containing user-defined tags. Differing from existing artist network visualization tools, the proposed prototype emphasizes commonalities as well as main differences between artist categorizations derived from user-defined tags, hence providing enhanced browsing experiences to users.
\end{abstract}

\section{Introduction}

One of the fastest growing media on the web is web-radio. There are now many web-radios available where millions of users spend a very significant amount of their time. Users can customize a million-track collection to very specific music tastes. Web-radios usually allow users to type in a tag that describes the music they want to hear (e.g. "acid jazz", "wake up", etc.), and music items with that tag will make up the personalized radio feed recommended to that user. Artist similarities (computed by methods described in Section 2) are also used to generate playlists. Albeit very useful, tag-based or similarity-based playlists are sometimes difficult for users to understand. The reason why a given artist or music was "selected" by the web radio is not always obvious and it can easily become confusing or frustrating for less experienced users that are unable to clearly express their musical preferences through queries. For example, very famous artists (e.g. "U2") can sometimes be considered "similar" to other -otherwise quite different- popular artists (e.g. "Queen", "Sting", "Coldplay", "Counting Crows"), just because they are also popular or end up receiving the same relatively uninformative tags (e.g. "pop").

In this paper, we explore the idea that networks of music artists contain rich and multifaceted information (music artist similarities, user tags, etc.) that can be useful for recommendations that go beyond the creation of playlists. We present RAMA, Relational Artist MAps, available through http://pattie.fe. up.pt/RAMA/ . RAMA is a visualization tool that allows the user to navigate inside the network of artists of the Last.fm web-radio. Our tool uses information about artists similarity and artists tags provided by Last.fm to produce a visualization of artists relations and corresponding user-defined tags in a graph, hence fostering the visualization of relational information. One of the original contributions of our work over Last.fm's web interface is to allow users not only to see which tags are common to a set of artists, but also those which are specific to a given artist when compared to similar artists.

\section{Related Work}

There are different approaches to music recommendation, which makr use of the different facets 
of music. Recommending music items is essentially based on the definition of a similarity metric between items, possibly tuned for a particular user. As developed in greater details in (Celma, 2006), this can be achieved by: (i) automatic analysis of contents (i.e. via algorithms computing e.g. rhythmic and tonal descriptions of audio files), (ii) expert analysis (as is the case of Pandora, or Tapestry ${ }^{1}$ ), (iii) collaborative filtering (i.e. exploiting a user-item relational matrix), (iv) co-occurrence analysis (using e.g. crawling techniques to fetch from the web text related to music artists and seek co-occurrences of artists names, terms, etc. (Schedl et al., 2007)), (v) analysis of meta-information provided by users (i.e. recommending music clips with a specific tag -e.g. "alternative rock"). Hybrid alternatives to these techniques also exist.

The most frequent way for systems to present music recommendations to users (e.g. on the Last.fm website), is by generating lists of potentially relevant music items (artist names, songs, etc.). An alternative way, is to provide users with more informative visualization methods for inspecting the similarities between music items (Pampalk et al., 2002), (Pampalk and Goto, 2007). For instance, a lot of attention has recently been given to the visualization of artist networks. In artist networks, two different types of data are usually presented. On the one hand, there is traditional encyclopedic data about individual artists. This includes biographic data such as names of albums, names of music tracks, photos and other images, etc. On the other hand, there is relational data, regarding e.g. artist similarities, connections between them, etc.

A popular metaphor for visualizing these two types of data is that of connected graphs, where the data is presented through nodes and edges connecting them. Connected graphs offer a number of "containers" to represent information (Shneiderman and Aris, 2006): (i) Node Labels, (ii) Node Attributes, (iii) Edge Labels, (iv) Edge Attributes (v) Edge Directions (either directed or undirected links). Given specific types of artist-related data one wants to visualize, a specific mapping must be done onto these data "containers". Nodes (or vertices) and edges are also central to the science of complex networks (Barabási, 2002), and a number of works in this field have recently brought some light onto the manifold intertwinements of musical artists networks (Cano et al., 2006), (Teitelbaum et al., 2008). There are several applications for visualizing artist networks as twodimensional connected graphs. For instance Musi-

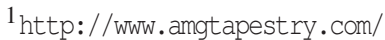

covery $^{2}$, TuneGlue's "music map"3, Gnod's "music map"4, Dimvision's "music map" music recommendation tool ${ }^{6}$, or the "SimilarArtistGraph"7 by Last.fm user Shoxrocks. These applications make use of both individual and relational data. In some applications, nodes can be expanded to reveal attributes such as label names, dates of album release, biographies and link to artist websites. In the abovementioned applications, data regarding the similarity between artists is gathered from third parties such as Last.fm or Amazon. Artists (i.e. nodes) that are somehow similar (i.e. that Last.fm considers similar, or that Amazon recommend together) share a link (i.e. are connected via an edge). A bird eye's view upon the topology of artist graphs may reveal clusters of artists, and users can embrace in one sight artists that are similar to their query as well as those similar to the answers, and so on.

Our system also uses third-party (i.e. Last.fm) similarity data to display music artists in 2D connected graphs, where edges represent similarities. In our prototype, node labels also convey similar data as they do. But an original aspect is that we intend to make further use of node attributes, as well as edge labels, edge attributes and directions, in order to convey more relational information (i.e. artist commonalities and specificities) computed from Last.fm data. By making use of more data, we sought a good balance between readability (avoiding cluttered use of space) and richness of the data presented to the user. Hence the special focus, in the design phase, on a proper use of graphical features (e.g. colors and transparencies) as well as interactivity between the user and the prototype (some information is shown by default, some other only as results of users' interactions).

\section{Last.FM}

Last.fm (http://www.last.fm ) is one of the leading internet-based social music platforms, where users can listen to music, find information about artists they like, or discover artists they might not know. Following Web 2.0 concepts, users can also set up their own profile, facilitating targeted automatic recommendations, among other things they can also get information about users with similar tastes, gigs

\footnotetext{
$2_{\text {http: //musicovery.com/ }}$

${ }^{3}$ http://audiomap.tuneglue.net/

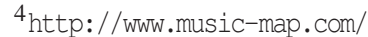

5 http://www.dimvision.com/musicmap/

${ }^{6}$ http://kylescholz.com/projects/speaking/ tae2006/music/

${ }^{7}$ http://lastfm. dontdrinkandroot.net/
} 
in their local area, videos, etc. Last.fm provides an interface for users to collaboratively edit encyclopedic information about artists. For some of the more popular artists there is also extensive biographic information already available.

User profiles -hence recommendations- are constantly updated via a software (free of use) which gathers ("scrobbles" in the Last.fm vernacular) statistics about the music listened to by users. User listening patterns are recorded and analyzed by Last.fm in order to better organize and recommend music. Users are also encouraged to organize the music they listen to by assigning tags to artists, or even to specific albums or tracks. The definition of tags is up to the users and can describe any aspect users believe are relevant, as music genres (e.g. "rock", "Viking metal"), locations (e.g. "Berlin"), mood (e.g. "chill"), opinions (e.g. "songs my mother would like"), contexts (e.g. "love") or just about anything that cross users' minds (see Figure 1 for examples of tags assigned to the band "Radiohead"). Tagging music helps users to browse Last.fm contents. But the real power of tags becomes clear when considering that tags of hundreds of thousands of users are combined, providing an emerging "bottom-up" categorization of music.

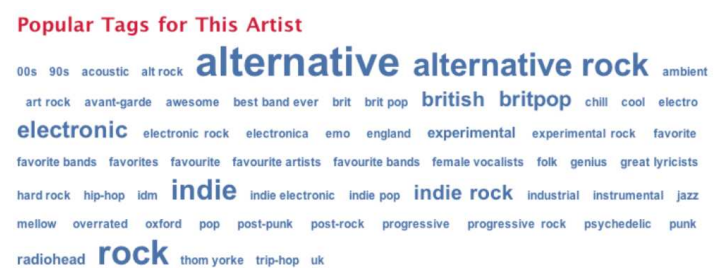

Figure 1: List of user tags for the band "Radiohead". Sizes of the letters correspond to popularity of the tag.

A cornerstone of Last.fm functionalities resides in links of similarity between artists (which can be seen on Figure 2 (a simple list), and which is central to automatic recommendations made to users). The algorithm used for computing similarities between artists is unknown -to the authors of this paper- but is probably based on (i) the analysis of user-added tags, (ii) user listening patterns such as co-occurrence statistics (users that listen to artist $\mathrm{X}$ also listen artist $\mathrm{Y}$ ), and (iii) user profiles information ("similar" users should like "similar" artists).

\section{RAMA: Relational Artist MAps}

RAMA is built on top of a client-server architecture. The visualization is performed on the client

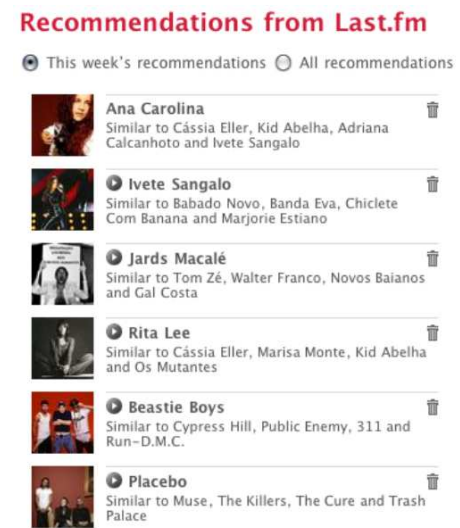

Figure 2: Personal artist recommendations made by Last.fm to one of the authors of this paper

side (the user application) using information obtained from the server via an HTTP request. The server manages the data that has been extracted from Last.fm site and performs all the necessary pre-processing operations to provide the client with the information needed for visualization.

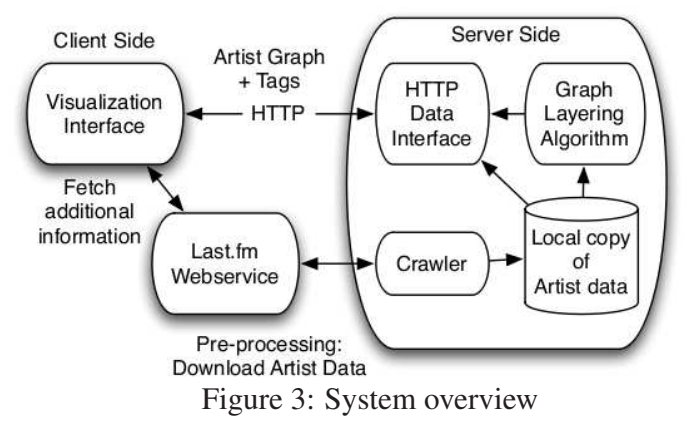

Given an initial query (i.e. an artist name) submitted by the user through a text-box in the client application, a request is sent to the server which provides all the information that the client application requires to draw the corresponding artist network. The network contains the artists that are found to be more similar to the queried artists, according to Last.fm's artist similarity index. It also contains the artists that are found by propagating the similarity query to the previously found ones so that we obtain artists several levels away from the initial artist. This allows the user to see the artist position in wider context (see e.g. Figure 4). The server provides the client with a list containing information regarding each node in the graph (i.e. each artist in the network), namely: (i) the $2 \mathrm{D}$ coordinates of the corresponding node in the graph, computed by our graph layering algorithm (see 
Section 4.2); (ii) the list of user-defined tags for each artist as taken from Last.fm site; (iii) explicit similarity relationships with other artists in the network (for drawing the necessary edges) as taken from Last.fm site. Once the visualization is rendered by the client application, users can either insert another query in the text-box, or browse through the network, interactively consulting tag information, and performing additional queries by clicking on the nodes. Interaction continues in this fashion until the user quits the application.

\subsection{Data Pre-processing}

Our system uses data that is freely available through Last.fm web-services $\mathrm{API}^{8}$. Through this web-service developers can access different data categories related to Last.fm users and media. Available data includes: (i) the profile of users of Last.fm, (ii) information and statistics related to artists, their albums and corresponding music tracks, (iii) tag information for each of the previous items, (iv) information about user created groups (e.g. fan groups), (v) information about message forums that users can create/participate, and (vi) geo-aware statistics about users and music preferences (more details can be found in the web service site). From all the data available, our system uses currently only data specially concerning artists, namely:

- basic artist data: name, URL of image, "popularity" index within Last.fm community,

- the list of the most similar artists for each artist. Last.fm assigns each similar artist a weight ranging from 100 (full similarity) to 0 (almost no similarity).

- information about the user-defined tags for each artist. Again, Last.fm assigns weights to quantify the association level for that tag: 100 means full association, while 0 indicates loose association.

Because each individual access to the Last.fm web service involves a considerable overhead due to network latency and server load, we created a local copy of the data we needed by crawling the webservice systematically. For a period of about a week (from January 30 to February 4 2008) we consulted Last.fm's web-service and obtained the previously described data for a total number of 583.000 artists. Local access to this data allowed to speed-up experimentation and to improve the global performance of our system. Although the Last.fm user community is constantly contributing and changing this information, we can assume that the relationships between

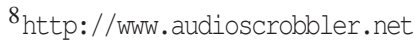

artists, which are the focus of our work, are more or less stable, at least for the reasonably popular artists. In any case, the crawling procedure can be repeated at any time in order to update data.

\subsection{Layering the Graph}

For layering the graph corresponding network of artists in a 2D plane, we implemented our own graph drawing system based on a force-directed placement strategy (Fruchterman and Reingold, 1991). Our implementation, in Perl, is completely integrated in the overall server-side framework which was also implemented in Perl. There are several parameters related to the graph layering operation that need to be set in order to create an appealing and useful visualization. The first parameter concerns the number of nodes (i.e. artists) that should be included in the graph. We wish to convey as much information as possible but we are limited to a given frame size. Layering graphs with many labeled nodes - which may have other attributes to be drawn- in a regular computer screen may generate rather confusing visualizations. Therefore we need to limit the number of nodes to a reasonably low value, for example between 15-50 nodes.

The second parameter has to do with the size of the context we wish to convey. Since we are limiting the number of nodes to a given maximum, there is a balance between (i) choosing more nodes that are closer to the initial artist, and thus providing more detail about the close relations of that artist, or (ii) choosing more nodes that are a few links away from the artist and thus provide a wider perspective about the artist and its relations. Our goal is to combine a good level of local detail with a wide enough perspective, and achieve a good balance between readability and richness of data presented to the user. We have defined 3 parameters to configure the properties of the network to be drawn around a given artist:

- level-1 branching factor: this parameter controls how many nodes directly connected to the initial one are to be visualized (the nodes chosen are the top similar ones).

- level-n branching factor: this parameter controls how every node in the graph except the initial one will branch.

- maximum branching distance: this parameter imposes a threshold on the maximum branching distance, i.e. how many links away can nodes be from the initial artist node.

With these parameters we perform an iterative expansion of the initial artist node, branching each node according to the corresponding factor. Increasing level- 
1 branching will promote local detail, while increasing level-n-branching and maximum branching distance will widen the context. In any case the number of nodes can never exceed a given pre-defined threshold.

\subsection{User Interface}

The user interface was developed in Processing ${ }^{9}$ and is responsible for generating the visualization and providing interactivity. Initially, the user can enter a name of an artist in a text-box. A query is then sent via HTTP to the server, which replies with the artist network data. For each artist (i.e. each node) in the network, the server sends back to the client the following data: (i) the name, (ii) a popularity index, (iii) the url of the photo of the artist in Last.fm server, (iv) the top 20 user-defined tags, (v) information about similarity with other artists (i.e. the node edges and their weights), and (vi) the coordinates for placing the corresponding node of the graph in a 2D plane. All this information is sent to the visualization interface in text format to allow a simple parsing procedure.

The interface application uses additional data that is directly fetched from Last.fm site at runtime, namely artist pictures and a short biographic description. For these media items performance constraints are not so severe (only two extra accesses for each artist in the network), so the interface can access that information directly as needed without requiring any intervention from the server.

On top of the basic network structure we place information related to user-defined tags. However, instead of presenting all the tags assigned to each artist over its corresponding node, we try and place the most common tags in the network in a such position that they will simultaneously describe all artists contained in a certain part of the network (simple animations of these tags permit to avoid readability problems). Such tag information is shown by default, no action being required from the user, allowing the user to easily identify the attributes that explain why certain artists are clustered in a specific part of the network.

For instance, in the network of "Radiohead" (see Figure 4), one can identify a region of artists tagged as "alternative" in the center because such a tag is common to most artists in the whole network. On the other hand, the region on the top-left branch is tagged "electronica", the common attribute of artists in that part of the network. Tag sizes are proportional to the number of artists for which they are relevant.

Moving the mouse cursor on top of a specific artist node results in the presentation of diverse data: the

\footnotetext{
${ }^{9}$ http://www.processing.org
}

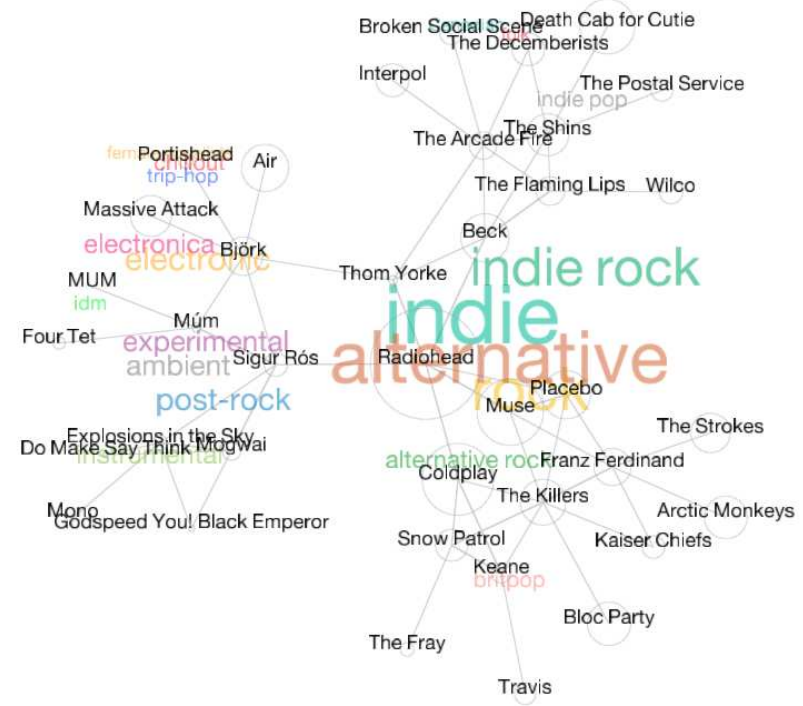

Figure 4: Example graph for artist "Radiohead".

artist picture, a short description and bio (gathered at run-time from the Last.fm site), and a link to its Last.fm webpage. Left-clicking on any artist name has the same effect as entering its name in the query box, i.e. sending a new query and refreshing the map with this query as seed. This allows simple user navigation through the artist network. To ensure that visualization always exhibits some degree of novelty, a new graph layout is computed in real time for each query, even if the query has been processed before.

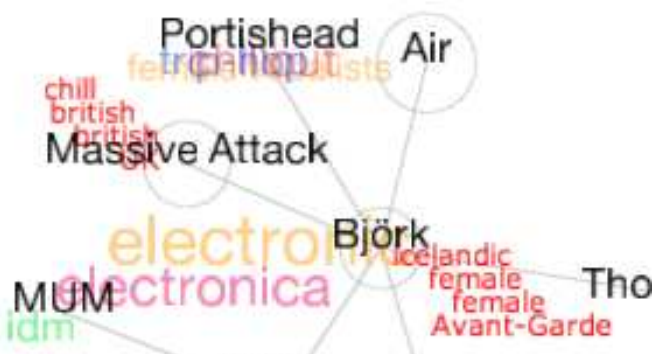

Figure 5: Examples of spećific tags for artists "Bjork" and "Massive Attack", both part of the "Radiohead" network.

An original feature of our user interface resides in the possibility to visualize tags that are specific to an artist in relation to another, i.e. those tags that are relevant to an artist but not to its neighbors. This information is shown only when the user crosses the mouse cursor over an edge. This action will result in the rendering of the tags that are specific to each 
of the two artists at their respective sides of the edge. In Figure 5 one can see an example of such a behavior. "Bjork" and "Massive Attack" are both part of the "Radiohead" network. Although they are both on a region characterized by the "electronic" tag, the tags "icelandic","female" and "avant-garde" are only assigned to "Bjork", while the tags "UK", "british" and "chill" are assigned only to "Massive Attack". This provides a very clear description of the unique features of each artist in the network. We believe this to be a very powerful tool in increasing user understanding about the network data. Table 3 shows a few more examples of tags that are found to be specific to a given artist ("Radiohead") when compared to other artists in its network.

\begin{tabular}{|l|l|}
\hline Radiohead & Sigur Ros \\
\hline $\begin{array}{l}\text { UK, pop, britpop, art } \\
\text { rock }\end{array}$ & $\begin{array}{l}\text { shoegaze, chillout, ice- } \\
\text { landic, ethereal }\end{array}$ \\
\hline Radiohead & Thom Yorke \\
\hline $\begin{array}{l}\text { progressive rock, 90s, } \\
\text { post-rock, experimental } \\
\text { rock }\end{array}$ & $\begin{array}{l}\text { radiohead, trip-hop, } \\
\text { electro, chillout, } \\
\text { singer-songwriter, male } \\
\text { vocalists }\end{array}$ \\
\hline Radiohead & Placebo \\
\hline $\begin{array}{l}\text { art rock, experimental, } \\
\text { UK }\end{array}$ & $\begin{array}{l}\text { emo, glam rock, metal, } \\
\text { punk }\end{array}$ \\
\hline Radiohead & Muse \\
\hline $\begin{array}{l}\text { electronica, art rock, } \\
\text { post-rock }\end{array}$ & progressive, emo, metal \\
\hline
\end{tabular}

Table 1: Pairwise discriminative tags between "Radiohead" and other similar artists.

\section{Conclusions and Future Work}

RAMA provides a simple yet efficient interface to navigate through the network of similar artists, allowing users to obtain a wider view about the artists they know, and to easily discover new bands and artists that they might like. It provides two simultaneous layers of information: (i) a graph built from artists and their connections, and (ii) overlaid labels containing userdefined tags that express the classification made by Last.fm community for each of the artists. From experimentation we have observed that the system effectively allows to identify clusters of tightly connected band and artists (such as for example former members of a band that pursued a solo career). Additionally, the visualization procedure in RAMA also emphasizes the main differences between artists, allowing the user also to visualize which are the most distinctive attributes of each artist.
Future work includes enhancing user experience by adding song snippets for each artist, so that the user can play them on demand while navigating across the network. Also, we plan to improve interactivity by allowing the user to optionally navigate though userdefined tags, and not just artists. We will also focus on allowing the user to manipulate the graph (zooming, rotating, etc) and to edit it. Editing capabilities will enable the user to remove nodes (artists) from the graph, expand only some, and thus generate a personalized graph, which could then be saved e.g. in the form of a playlist.

\section{Acknowledgments}

This work was partially supported by grant SFRH/BD/ 23590/ 2005 from FCT (Portugal), cofinanced by POSI. Thanks to Elias Pampalk from Last.fm for insightful comments.

\section{REFERENCES}

Barabási, A. (2002). Linked: The New Science of Networks. Perseus Publishing.

Cano, P., Celma, O., Koppenberger, M., and Buldú, J. (2006). The topology of music recommendation networks. Chaos, 16(013107).

Celma, O. (2006). Music recommendation: A multi-faceted approach. Master's thesis, Universitat Pompeu Fabra, Barcelona.

Fruchterman, T. M. J. and Reingold, E. M. (1991). Graph drawing by force-directed placement. Softw. Pract. Exper., 21(11):1129-1164.

Pampalk, E. and Goto, M. (2007). MusicSun: A new approach to artist recommendation. In Proceedings of the International Conference on Music Information Retrieval.

Pampalk, E., Rauber, A., and Merkl, D. (2002). Contentbased Organization and Visualization of Music Archives. In Proceedings of the ACM Multimedia, pages 570-579, Juan les Pins, France. ACM.

Schedl, M., Knees, P., Widmer, G., Seyerlehner, K., and Pohle, T. (2007). Browsing the Web Using Stacked Three-Dimensional Sunbursts to Visualize Term CoOccurrences and Multimedia Content. In Proceedings of the 18th IEEE Visualization Conference.

Shneiderman, B. and Aris, A. (2006). Network visualization by semantic substrates. IEEE Transactions on Visualization and Computer Graphics, 12(5).

Teitelbaum, T., Balenzuela, P., Cano, P., and Buldú, J. (2008). Community structures and role detection in music networks. Chaos, 18(043105). 